\title{
Principais causas de mortalidade hospitalar de idosos no Estado do Pará, Brasil
}

\author{
Matheus Sodré de Araújo*, Ana Karoline Brito de Oliveira*, Israel Júnior Borges do Nascimento**, \\ Katiane da Costa Cunha**
}

\section{Resumo}

Globalmente, percebe-se um envelhecimento populacional, fato que interfere diretamente em políticas de saúde direcionadas aos idosos, visto que a senescência é inevitável e está ligada principalmente ao surgimento de doenças crônicas não transmissíveis. No Brasil, o processo de enveIhecimento é acompanhado de fatores que interferem na qualidade de vida do idoso, consequentemente na morbimortalidade hospitalar. Nesse sentido, o objetivo desse estudo foi analisar as principais causas de mortalidade de idosos hospitalizados no estado do Pará, no período de janeiro de 2008 a agosto de 2019. Trata-se de um estudo epidemiológico transversal descritivo de série temporal, com dados de idosos disponíveis no Sistema de Informações Hospitalares do Sistema Único de Saúde (SIH/SUS). Para análise de dados foi utilizado o programa BioEstat ${ }^{\circledR}$ 5.3. Verificou-se que dentre as unidades federativas da Região Norte, o estado do Pará apresenta a maior porcentagem de óbitos de idosos $(41,4 \%)$. Idosas com faixa etária $\geq$ a 80 anos $(35,3 \%)$ tem o maior percentual de óbitos no sexo feminino, enquanto óbi- tos são mais frequentes em homens com faixa etária de 70 a 79 anos (36,5\%). As doenças do aparelho circulatório $(27,7 \%)$ são a principal causa de mortalidade de idosos, seguida das doenças do aparelho respiratório $(22,2 \%)$. Na análise por sexo, destacam-se como principais causas de óbito as doenças endócrinas, nutricionais e metabólicas em indivíduos do sexo feminino $(4,5 \%)$ e as doenças do aparelho digestivo no sexo masculino (8,3\%). Patologias do aparelho circulatório e sistema respiratório são responsáveis pela maior fração de óbitos em pacientes idosos no estado do Pará. Esse dado encontra-se em consonância ao perfil de mortalidade observado em outras regiões do país, para o mesmo grupo etário. Políticas de saúde pública, baseadas na prevenção e promoção em saúde, assim como ampliação a acesso a serviços assistenciais médicos tornam-se fundamentais para a garantia de um enveIhecimento saudável.

Palavras-chave: Mortalidade Hospitalar; Saúde do idoso; Causas de morte; Sistema Único de Saúde.

* Dicente do Curso de Medicina. Universidade do Estado do Pará, Campus VIII/Marabá. Av. Hiléia, Agrópolis do Incra s/n - Amapá, Marabá - PA, 68502-100.

** Médico. Universidade Federal de Minas Gerais. Av. Pres. Antônio Carlos, 6627 - Pampulha, Belo Horizonte - MG, 31270-901.

**** Pós Doutora em Teoria e Pesquisa do Comportamento. Professora do Curso de Medicina. Universidade do Estado do Pará, Campus VIII/Marabá. Av. Hiléia, Agrópolis do Incra s/n - Amapá, Marabá - PA, 68502-100.

http://dx.doi.org/10.5335/rbceh.v17i1.10655 


\section{Introdução}

Desde 1950, percebe-se uma modificação estrutural na composição etária das populações em todo o mundo. O expressivo aumento demográfico e de expectativa de vida resultou em uma profunda alteração do perfil de atividades laborais, socioeconômicas e organizacionais. Como aspecto resultante secundário a essas transformações, ressaltam-se as mudanças substanciais no perfil de mortalidade em diferentes idades e estágios econômicos, especialmente relacionado ao desenvolvimento sanitário e médico na maioria dos países do mundo (BLOOM, 2011). Em momento primário, anterior ao período da transição epidemiológica e demográfica, mais indivíduos eram acometidos por doenças infectocontagiosas, como consequência de condições neonatais precárias e desnutrição, além de baixa instrução e acesso a premissas de saneamento básico. Entretanto, atualmente, doenças crônicas não transmissíveis (doenças crônico-degenerativas) possuem maior repercussão sistemática no sistema de saúde de países desenvolvidos e em desenvolvimento, e são adicionalmente agravadas e vinculadas ao envelhecimento populacional (BEARD et al., 2016).

Reconhece-se que o envelhecimento é um processo natural, dinâmico e progressivo, em que se espera que ocorram mudanças físicas, psicológicas e fisiológicas, ambas contínuas, até comprometimento global do organismo (FREITAS, 2013). No Brasil, o pro- cesso de envelhecer vincula-se a uma série de condutas negligentes, as quais são fomentadas pela falta de políticas públicas assistenciais, que interferem diretamente na qualidade de vida do idoso. Entre elas, ressaltam-se condições econômicas insuficientes para a sua subsistência, moradias inadequadas, diminuição de práticas assistencialistas às populações socialmente vulneráveis, assim como o cancelamento de programas existentes e a ausência de saneamento básico (SANTOS; LIMA JÚNIOR, 2014). Outrossim, o Sistema Único de Saúde (SUS) apresenta falhas reparáveis, tais como a dificuldade da integração dos serviços nos diferentes níveis de atenção e baixa oferta de atendimento domiciliar (CORDEIRO; MARTINS, 2018), os quais resultam em índices elevados de doenças crônicas e elevadas internações hospitalares da população idosa (PARENTE, 2018).

Nesse âmbito de interesse, a Autorização de Internação Hospitalar (AIH) é um instrumento registrador regulatório do atendimento hospitalar pelo SUS, na qual é digitalizada pelo Sistema de Captação da Internação que, posteriormente, é enviada para o Sistema de Informação Hospitalar (SIH/SUS). Baseado nos registros supracitados e interligados ao SIH/SUS, consolida-se a criação e manutenção do Banco de Dados Nacional, o qual é utilizado pelos gestores de saúde e tomadores de decisão para controle, avaliação e auditoria de suas gestões. Dessa forma, o SIH/SUS torna-se um importante banco dados da morbidade hospitalar, sendo possível comparar, de 
forma sistemática, a morbimortalidade em diferentes regiões do país. Nessa perspectiva, o óbito hospitalar é utilizado indiretamente como um dos indicativos de desempenho clínicos em vários países, sendo ainda base de estudos secundários que avaliam fatores associados ao óbito, como tempo de internação, perfil de gravidade, entre outras questões (CORDEIRO; MARTINS, 2018; MCCORMICK; PEARSON; WHITE, 2015).

Uma forma de identificar as causas de óbito é por meio da lista de morbidade dos idosos da Classificação Internacional de Doenças (CID-10), ferramenta criada com o objetivo de padronizar e catalogar as doenças e problemáticas relacionadas à saúde baseado na Nomenclatura Internacional de Doenças determinada pela Organização Mundial da Saúde (DEPARTAMENTO DE INFORMÁTICA DO SUS, [s.d.]). Em estudos anteriores das regiões Nordeste, Sul e Sudeste brasileiras, sabe-se que as principais causas de mortes de idosos são aquelas associadas o aparelho circulatório, doenças do aparelho respiratório e neoplasias (JÚNIOR; LOFFREDO, 2014; KERNKAMP,2016; PARENTE,2018).

No Estado do Pará, o único sistema de notificação que trata da mortalidade hospitalar de idosos é o SIH/SUS. Dessa forma, evidencia-se a escassez de estudos com temáticas relacionadas às causas dos óbitos hospitalares da população idosa no Estado do Pará, Brasil. Assim, o objetivo desse estudo foi avaliar as principais causas de mortalidade hospitalar de idosos, para ambos os sexos, no estado do Pará.

\section{Métodos}

Trata-se de um estudo transversal descritivo de série temporal, associados aos registros de óbito em idosos no Brasil, coletados na plataforma do SIH/SUS e secundários de AIHs. Ressalta-se que os aspectos de identificação dos pacientes não são realizados, fator dispensador de aprovação do Comitê de Ética em Pesquisa, segundo a Resolução do Conselho Nacional de Saúde no 466/12. Ademias, esse estudo foi fundamentado na declaração Garther, que delimita as melhores condutas para relatos de estudos que quantificam a saúde de populações e seus determinantes por meio da síntese de informações de variadas fontes (STEVENS et al., 2017).

Foram coletados dados referentes aos óbitos de idosos referentes ao período de janeiro de 2008 até julho de 2019, na abrangência geográfica Brasil e Pará, que estavam disponibilizados integralmente no SIH/SUS por meio do DATASUS. Para avaliação de nível nacional, fixou-se a coluna "Faixa Etária", a qual engloba campos selecionáveis de perfil etário (60 a 69, 70 a 79 e $\geq$ de 80 anos), e não se fixou a categoria associada, variando entre Região, Região/ Unidade da Federação, Sexo e Cor/ Raça. Para análise focalizada (Estado do Pará), fixou-se a coluna "Faixa Etária" 1, e não se fixou a categoria linha associada, variando entre Região de Saúde (CIR), Capítulo CID - 10. A lista de tabulação de causas para os óbito em idosos foram baseadas nas Notas técnicas do Tabnet DATASUS. 
Para avaliação estatística, definiu-se comparar dados do Brasil, da Região Norte, e do Estado do Pará. Utilizou-se o Software BioEstat@ versão 5.3. Adicionalmente, a tabulação e análise dos dados ocorreram por meio do programa TABWIN® e Microsoft Excel ${ }^{\circledR}$. O programa QGIS® foi utilizado na confecção e edição do mapa. Para análise dos dados, considerou-se sempre o índice de significância de $95 \%$ ( $\mathrm{p}<0,05)$. A taxa de mortalidade apresentada corresponde à razão entre a quantidade de óbitos e o número de AIH aprovadas, computadas como internações, no período, multiplicada por 100. Utilizou-se o teste qui-quadrado para avaliar o valor de dispersão entre os grupos avaliados $\left(\chi 2=\Sigma\left[(0-\mathrm{e})^{2} / \mathrm{e}\right]\right.$, sendo que "o" é a frequência observada para cada classe e "e" representa a frequência esperada para aquela classe). Para essa análise, considerou-se a relação entre óbitos entre diferentes estados da região norte, entre as faixas etárias de idosos (60 a 69 anos, 70 a 79 anos e $\geq$ a 80 anos) e entre as causas de óbito de acordo com o sexo dos idosos.

\section{Resultados}

De forma global, o valor absoluto de internações de idosos com altas por óbito no Brasil nos períodos de janeiro de 2008 a agosto de 2019 é 3.344.767 milhões de um total de 5.190.878 milhões, o equivalente a $64,4 \%$ de óbitos. Baseado em uma perspectiva regional de valor absoluto de óbitos, em ordem decrescente, tem-se a Região Sudeste possui 1.665.209 registros, seguida das Regiões Nordeste (701.886), Sul (646.082), Centro-oeste (194.515) e Norte (137.075). Relacionado a frequência de óbitos focalizada na Região Norte, sumarizamos na Tabela 1 a evolução dos registros nacionais de janeiro de 2008 a agosto de 2019 .

Tabela 1: Caracterização de idosos que evoluíram à óbito e taxa de mortalidade (TM) segundo a faixa etária, Região Norte, janeiro de 2008 - agosto de 2019. (n=137.688)

\begin{tabular}{|c|c|c|c|c|c|c|c|c|c|c|c|}
\hline \multirow{3}{*}{$\begin{array}{l}\text { Região } \\
\text { Norte }\end{array}$} & \multicolumn{10}{|c|}{ Idade (em anos) } & \multirow{3}{*}{$p$-valor } \\
\hline & \multicolumn{3}{|c|}{60 a 69} & \multicolumn{3}{|c|}{70 a 79} & \multicolumn{3}{|c|}{ Maior ou igual a 80} & \multirow{2}{*}{$\begin{array}{c}\text { Total } \\
\mathrm{n} \\
\end{array}$} & \\
\hline & $\mathrm{n}$ & $\%$ & $\mathrm{TM}^{*}$ & $\mathrm{n}$ & $\%$ & $\mathrm{TM}^{*}$ & $\mathrm{n}$ & $\%$ & $\mathrm{TM}^{*}$ & & \\
\hline Pará & 18841 & 44,0 & 5,5 & 20246 & 42,6 & 7,6 & 17996 & 39,1 & 11,1 & 57083 & \\
\hline Amazonas & 8151 & 19,0 & 7,5 & 8833 & 18,6 & 10,4 & 8813 & 19,1 & 15,7 & 25797 & \\
\hline Rondônia & 5489 & 12,8 & 6,0 & 6648 & 14,0 & 8,5 & 6426 & 14,0 & 13,3 & 18563 & \\
\hline Tocantins & 4856 & 11,3 & 6,5 & 5871 & 12,4 & 8,8 & 6605 & 14,3 & 14,2 & 17332 & \\
\hline Acre & 2604 & 6,1 & 8,3 & 3033 & 6,4 & 11,9 & 3378 & 7,3 & 18,2 & 9015 & $\rho<0,0001$ \\
\hline Amapá & 1728 & 4,0 & 8,9 & 1778 & 3,7 & 11,8 & 1795 & 3,9 & 17,7 & 5301 & \\
\hline Roraima & 1547 & 3,6 & 7,9 & 1526 & 3,2 & 11,1 & 1524 & 3,3 & 16,7 & 4597 & \\
\hline Total & 43216 & 100,0 & 6,3 & 47935 & 100,0 & 8,7 & 46537 & 100,0 & 13,3 & 137688 & \\
\hline
\end{tabular}

Fonte: Sistema de Informações Hospitalares do SUS (SIH-SUS) - BRASIL, DATASUS, 2019.

Nota: *TM: Taxa de mortalidade por cada 100 AIH aprovadas. 
A análise do valor absoluto, nas diferentes faixas etárias e nas diferentes unidades federativas da Região Norte, mostra o estado do Pará como o primeiro em número global de óbitos de idosos. Contudo, o estado do Amapá registrou a maior taxa de mortalidade $(8,9)$ na faixa etária de 60 a 69 anos, enquanto o estado do Acre tem maior índice nas faixas etárias de 70 a 79 anos $(11,9)$ e $\geq$ a 80 anos (18,2). Ademais, observa-se que na Região Norte, há maior número absoluto de óbitos no grupo etário entre 70 a 79 anos (47.935), enquanto a faixa etária $\geq$ a 80 anos de idade tem a maior taxa de mortalidade entre as faixas etárias avaliadas $(13,3)$.

Considerando-se especificamente o estado do Pará, a Figura 1 evidencia em detalhe a frequência de óbitos de idosos em cada uma das micro-regiões de saúde paraenses.

Figura 1: Número de óbitos, em valor absoluto, de idosos nas regiões de saúde do estado do Pará $(n=57.083)$. Janeiro de 2008- agosto de 2019

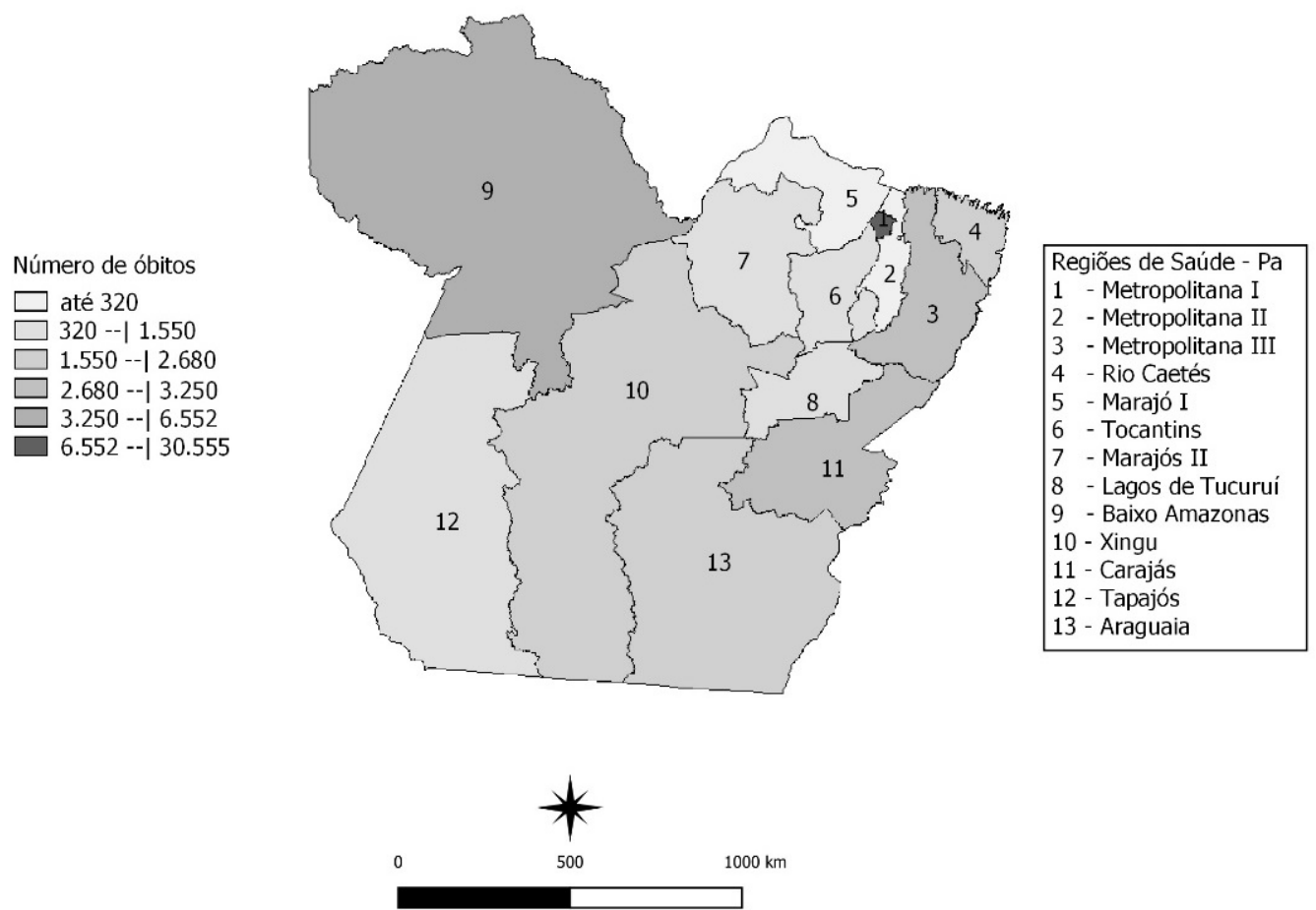

Fonte: Sistema de Informações Hospitalares do SUS (SIH-SUS) - BRASIL, DATASUS, 2019. 
De acordo com a Figura 1, as quatro regiões com maiores números de óbito em valor absoluto são: Metropolitana I (30.555), Baixo Amazonas (6.551), Metropolitana III (3.247) e Carajás (3.182). Além disso, percebe-se que as regiões Metropolitana I e Baixo Amazonas encontram-se acima da média regional de óbitos, considerando-se as diferentes micro-regiões. As regiões Metropolitana III e Carajás encontram-se próximas da média regional de óbito, considerando-se todas as causas de mortalidade.

Referente a taxa de mortalidade por sexo nos óbitos de idosos no Pará, observa-se que no sexo feminino, a faixa etária $\geq$ a 80 anos possui o maior percentual de óbitos $(35,3 \%)$ e maior taxa de mortalidade $(11,1)$. Nos indivíduos do sexo masculino, a faixa etária de 70 a 79 anos de idade tem o maior percentual de óbitos (36,5\%) e enquanto a faixa etária de $\geq$ a 80 anos possui maior taxa de mortalidade (11,1). Dessa forma, em relação ao percentual total de óbitos, indivíduos do sexo feminino evoluem ao óbito mais tardiamente que aqueles do sexo masculino, quando internados em ambiente hospitalar. A descrição detalhada do perfil de óbitos por grupo etário no Estado do Pará encontra-se na Tabela 2.

Tabela 2: Caracterização de idosos que evoluíram à óbito e taxa de mortalidade (TM) de acordo com o sexo no estado do Pará (PA). Janeiro de 2008 - agosto de 2019. (n= 57.083)

\begin{tabular}{|c|c|c|c|c|c|c|c|}
\hline \multirow{2}{*}{$\begin{array}{c}\text { Faixa etária } \\
\text { (anos de idade) }\end{array}$} & \multicolumn{3}{|c|}{ Feminino } & \multicolumn{3}{|c|}{ Masculino } & \multirow{2}{*}{$p$ - valor } \\
\hline & $\mathrm{n}$ & $\%$ & $\mathrm{TM}^{*}$ & $\mathrm{n}$ & $\%$ & $\mathrm{TM}^{*}$ & \\
\hline 60 a 69 & 7958 & 30,4 & 5,0 & 10883 & 35,2 & 5,9 & \multirow{4}{*}{$p<0,0001$} \\
\hline 70 a 79 & 8960 & 34,3 & 7,1 & 11286 & 36,5 & 8,0 & \\
\hline Maior ou igual a 80 & 9233 & 35,3 & 11,1 & 8763 & 28,3 & 11,1 & \\
\hline Total & 26151 & 100 & & 30932 & 100 & & \\
\hline
\end{tabular}

Fonte: Sistema de Informações Hospitalares do SUS (SIH-SUS) - BRASIL, DATASUS, 2019

*TM = Taxa de mortalidade por cada $100 \mathrm{AIH}$ aprovadas.

Avaliando-se o tempo de permanência no hospital (período transcorrido entre a admissão e o óbito), indivíduos do sexo masculino associam-se a um maior número de dias de internação (2.310.566) em relação ao sexo feminino (1.968.348 dias). Contudo, existe uma variação nessa frequência quando a análise é feita por faixas etárias. A faixa etária entre 60 e 69 anos evidencia maior número de dias de internação hospitalar, sendo o tempo de permanência do sexo masculino em dias (1.062.184) maior do que o do sexo feminino (825.472 dias), assim como na faixa etária entre $70 \mathrm{e}$ 79 anos, a qual mantém o predomínio do sexo masculino (810.846 dias) em relação ao sexo feminino (686.042 dias). 
Entretanto, em indivíduos com mais de ças do aparelho circulatório (15.837), X80 anos, o tempo de internação do sexo doenças do aparelho respiratório (12.683) feminino em dias (456.834) é superior ao do sexo masculino (437.536 dias).

e I - algumas doenças infecciosas e

As principais causas de óbito, de acordo com a Classificação CID-10, são aquelas associadas ao capítulo IX - doenparasitárias (8.629), em ambos os sexos, em idosos no Pará. A descrição detalhada das principais causas de óbitos de idosos paraenses encontra-se na Tabela 3.

Tabela 3: Causas de óbitos em idosos segundo os critérios do CID - 10 no estado do Pará (PA). Janeiro de 2008 - agosto de 2019. ( $n=57.083)$

\begin{tabular}{|c|c|c|c|c|c|c|c|}
\hline \multirow{2}{*}{$\begin{array}{l}\text { Capítulo } \\
\text { CID - } 10\end{array}$} & \multicolumn{2}{|c|}{ Feminino } & \multicolumn{2}{|c|}{ Masculino } & \multicolumn{2}{|c|}{ Total } & \multirow{2}{*}{ p-valor } \\
\hline & $\mathrm{n}$ & $\%$ & $\mathrm{n}$ & $\%$ & $\mathrm{n}$ & $\%$ & \\
\hline I & 4157 & 15,9 & 4472 & 14,5 & 8629 & 15,1 & \\
\hline II & 2161 & 8,3 & 2601 & 8,4 & 4762 & 8,3 & \\
\hline III & 220 & 0,8 & 230 & 0,7 & 450 & 0,8 & \\
\hline IV & 1173 & 4,5 & 1026 & 3,3 & 2199 & 3,9 & \\
\hline V & 5 & 0,0 & 10 & 0,0 & 15 & 0,0 & \\
\hline VI & 339 & 1,3 & 388 & 1,3 & 727 & 1,3 & \\
\hline VII & 0 & 0,0 & 2 & 0,0 & 2 & 0,0 & \\
\hline VIII & 2 & 0,0 & 0 & 0,0 & 2 & 0,0 & \\
\hline IX & 7267 & 27,8 & 8570 & 27,7 & 15837 & 27,7 & \\
\hline$x$ & 5902 & 22,6 & 6781 & 21,9 & 12683 & 22,2 & \\
\hline XI & 1890 & 7,2 & 2570 & 8,3 & 4460 & 7,8 & 0 \\
\hline XII & 203 & 0,8 & 237 & 0,8 & 440 & 0,8 & ו \\
\hline XIII & 41 & 0,2 & 33 & 0,1 & 74 & 0,1 & \\
\hline XIV & 1338 & 5,1 & 1862 & 6,0 & 3200 & 5,6 & \\
\hline$X V$ & 2 & 0,0 & 0 & 0,0 & 2 & 0,0 & \\
\hline XVI & 8 & 0,0 & 13 & 0,0 & 21 & 0,0 & \\
\hline XVII & 24 & 0,1 & 17 & 0,1 & 41 & 0,1 & \\
\hline XVIII & 531 & 2,0 & 738 & 2,4 & 1269 & 2,2 & \\
\hline XIX & 726 & 2,8 & 1173 & 3,8 & 1899 & 3,3 & \\
\hline$X X$ & 2 & 0,0 & 1 & 0,0 & 3 & 0,0 & \\
\hline$X X I$ & 160 & 0,6 & 208 & 0,7 & 368 & 0,6 & \\
\hline Total & 26151 & 100,0 & 30932 & 100,0 & 57083 & 100,00 & \\
\hline
\end{tabular}

Fonte: Sistema de Informações Hospitalares do SUS (SIH-SUS) - BRASIL, DATASUS, 2019. 
No sexo feminino, verificou-se que os capítulos III - doenças do sangue e dos órgãos hematopoiéticos e alguns transtornos imunitários $(0,8 \%)$, IV - doenças endócrinas e metabólicas $(4,5 \%)$ e XIII - doenças do sistema osteomuscular e do tecido conjuntivo $(0,2 \%)$ mostraram maiores percentuais $(5,5 \%)$ do que no sexo masculino $(4,1 \%)$. Por outro lado, considerando-se o sexo masculino, os capítulos II - neoplasias $(8,40 \%)$, XI Doenças do aparelho digestivo (8,30\%), XIV - doenças do aparelho geniturinário $(6,0 \%)$, XVIII - sintomas, sinais e achados anormais de exames clínicos e de laboratório, não classificados em outra parte $(2,40 \%)$, XIX - lesões, envenenamento e algumas outras consequências de causas externas $(3,80 \%)$ e XXI - fatores que influenciam o estado de saúde e $o$ contato com os serviços de saúde $(0,70 \%)$ possuem maiores percentuais $(29,6 \%)$ do que o sexo feminino (26\%).

\section{Discussão}

Com base nos resultados encontrados, as principais causas de morte de idosos, em ambos os sexos, no estado do Pará, são associadas às doenças do aparelho circulatório e as doenças do aparelho respiratório. $\mathrm{O}$ sexo feminino, em relação ao masculino, se destaca com uma maior frequência relativa de casos de doenças endócrinas e metabólicas. Contrariamente, no sexo masculino, há maior frequência relativa de casos de doenças do aparelho digestivo. Ademais, considerando o valor absoluto, mulheres idosas evoluem à óbito mais tardiamente que os homens idosos. Em relação ao período entre o tempo de internação até o momento do óbito, os homens permanecem hospitalizados por mais tempo quando comparados as mulheres.

As doenças do aparelho circulatório, apontadas como as principais causas da mortalidade de idosos no Pará, encontra-se em consonância com os resultados de estudos mundiais. Em 2010, as cardiovasculopatologias foram vinculadas as principais causas de morte no mundo (LOZANO et al., 2012). De forma similar, estudos brasileiros (2008/2009) sugeriram que a mortalidade de idosos que referiam doenças do coração foi $76 \%$ maior do que aqueles que não referiam esses problemas (BORIM; FRANCISCO; NERI, 2017). Ademais, de acordo com dados do Estado do Rio Grande do Norte, em 2014, as doenças do aparelho circulatório foram o principal motivo da mortalidade dos idosos com 60 a 69 anos $(32,76 \%)$ e com 80 anos e mais $(35,26 \%)$ (OLIVEIRA; MEDEIROS; LIMA, 2015).

Ainda que na Região Sudeste as doenças do aparelho circulatório tenham reduzido nos últimos anos - a exemplo de Marília/SP, cujos idosos com mais de 60 anos reduziram $0,05 \%$ no sexo masculino e 20,38\% no sexo feminino (CARVALHO et al., 2014)-, nas Regiões Norte e Nordeste esse perfil de mortalidade aumentou nos últimos anos. Melhora no processo diagnóstico e na investigação da causa de morte, aumento na urbanização, piora das condições socioeconômicas das regiões e baixa escolaridade podem ser possíveis justificativas para esse 
aumento nessas duas macrorregiões brasileiras (MANSUR et al., 2006).

Considerando-se os mecanismos fisiopatológicos associados ao envelhecimento e manifestações no aparelho cardiovascular, diferentes processos podem ser citados. Redução da distensibilidade arterial, redução do tamanho das fibras musculares cardíacas, diminuição do débito cardíaco, diminuição da frequência respiratória, aumento da pressão sistólica arterial, aumento do colesterol total no sangue podem ser mecanismos importantes que resultam em maior acometimento desse sistema (TORTORA; DERRICKSON, 2016).

A segunda maior causa de mortalidade de idosos no Estado do Pará, no período analisado, foi vinculada às doenças do aparelho respiratório. De forma similar, em estudo longitudinal no Estado de São Paulo, evidenciou que as doenças do aparelho respiratório são também a segunda principal motivação de óbitos, com aumento no período de $41,8 \%$ para o sexo masculino e 38,3\% para o sexo feminino (TELAROLLI JÚNIOR; LOFFREDO, 2014). Por outro lado, no Rio Grande do Norte, a morte de idosos por doenças do aparelho respiratório foi a quinta principal causa na faixa etária de 60 a 69 e terceira principal causa na faixa etária de 80 anos e mais (OLIVEIRA; MEDEIROS; LIMA, 2015). Ademais, em Marília/SP, as doenças do aparelho respiratório foram a terceira principal causa da mortalidade dos idosos, tendo na faixa etária acima ou igual a 80 anos um aumento da mortalidade de $39,31 \%$ para o sexo masculino e $57,92 \%$ para o sexo feminino (CARVALHO et al., 2014). As diferentes prevalências de doenças do aparelho respiratório nas diversas regiões do Brasil podem estar relacionadas aos focos de queimadas e o nível de umidade do ar em alguns períodos do ano como constatado em um estudo realizado no estado de Rondônia sobre asma (RODRIGUES et al., 2010). Além disso, a susceptibilidade imunológica do envelhecimento pode estar ligada aos dados expressivos relacionados a esse grupo de doenças (OLIVEIRA; MEDEIROS; LIMA, 2015).

Doenças metabólicas foram a quarta causa principal de mortalidade de idosos do sexo feminino no Pará. Em São Paulo, as doenças endócrinas, nutricionais e metabólicas também ocuparam a quarta posição de óbitos de indivíduos do sexo feminino, sendo que em 2010, correspondia a $6,4 \%$ do total de óbitos acima dos 60 anos (MENDES, 2012).

Alterações do eixo Hipotálamo-Hipófise-Glândulas, o qual envolve as principais glândulas do organismo na manutenção da homeostasia humana, parecem estar afetados em diferentes momentos do envelhecimento (TEIXEIRA et al., 2019; TORTORA; DERRICKSON, 2016).

Em relação à mortalidade de idosos do sexo masculino no Pará, as doenças do aparelho digestivo são significativas. Segundo dados do Ministério da Saúde sobre morbidade masculina no Brasil, a quarta maior causa de óbitos do sexo masculino são os problemas de aparelho digestivo. Ademais, $88 \%$ dos óbitos são diretamente ou indiretamente provo- 
cados por doenças alcoólicas (consumo frequente de álcool) (MINISTÉRIO DA SAÚDE, 2017). Esse cenário condiz com a incidência de internações masculinas vinculadas ao consumo de álcool, e consequentemente maior índice de doenças do aparelho digestivo, incluindo as doenças hepáticas (MENDES; SALA, 2011). Sabe-se que o consumo excessivo de álcool gera quadros agudo e crônico de alcoolismo, os quais podem gerar efeitos no sistema digestivo (por alterações hepáticas e gástricas) e psiquiátricas graves (KUMAR; ABBAS; ASTER, 2016). Interessantemente, em um estudo sobre a mortalidade de idosos de São Paulo, a mortalidade proporcional por conta de doenças do aparelho digestivo no sexo masculino $(5,7)$ é maior do que no sexo feminino $(4,8)$ (MENDES, 2012).

Em nosso estudo verificou-se que mulheres do Estado do Pará possuem maior longevidade quando comparadas a homens do mesmo Estado. Em função das mulheres serem mais assíduas no acesso aos serviços de saúde, pela maior prevalência de determinadas doenças no sexo masculino, assim a insuficiência de programas de saúde especializados na saúde masculina, podem ser justificativas associadas a esse perfil observado (LAURENTI; JORGE; GOTLIEB, 2005). Ademais, têm-se relatado que homens possuem menor interesse na busca do serviço de saúde, assim como não efetivam a correta adesão a tratamentos prescritos (MINISTÉRIO DA SAÚDE, 2017). Contudo, o indivíduo não pode ser totalmente culpabilizado pela tendência observada, sendo que outros fatores possam contribuir para o cenário da saúde masculina. Estudos sobre a Política Nacional de Atenção Integral à Saúde do Homem, revelaram que existem deficiências, preconceitos e lacunas na formação dos profissionais de saúde para o acolhimento de indivíduos do sexo masculino (MOREIRA; GOMES; RIBEIRO, 2016). Acerca do tempo de internação (em que homens possuem maior tempo de internação quando comparados a mulheres), resultados com idosos residentes de Recife/PE, evidenciaram tendência amostral similar a encontrada em nosso estudo (MOROSINI et al., 2011).

\section{Conclusão}

Os achados desse estudo permitem concluir que as doenças do aparelho circulatório são a principal causa de morte de idosos no Estado do Pará, seguida das doenças do aparelho respiratório. Ressalta-se que existem diferenças entre as principais doenças que provocam a morte entre os sexos masculino e feminino. Além disso, indivíduos do sexo feminino percorrem maior intervalo de tempo para evoluírem ao óbito quando comparados aos homens. Adicionalmente, indivíduos do sexo masculino permanecem mais tempo hospitalizados até evoluírem ao óbito. As informações ressaltadas nesse estudo podem ser úteis para a implementação de políticas públicas vinculadas a saúde do idoso no Estado do Pará. 


\section{Main causes of in-hospital mortality among elderly patients in Pará, Brazil}

\section{Abstract}

Globally, it is perceived the populational aging, which influences directly in health policies aimed elderly individuals, since senescence is inevitable and is linked mainly to the emergence of non-communicable diseases. In Brazil, the aging process is accompanied by factors that interfere within the quality of life of elderly patients, and as a consequently in hospital morbidity and mortality. In this perspective, we aimed to analyze the main causes of in-hospital mortality among elderly patients in the State of Pará (Brazil), from January 2008 to August 2019. This is a descriptive cross-sectional epidemiological study of time series, with data on elderly people available in the Sistema de Informações Hospitalares do Sistema Único de Saúde $(\mathrm{SIH} / \mathrm{SUS})$. For data analysis, the BioEstat ${ }^{\circledR}$ 5.3 program was used. It was found that amongst different states in the Northern Region of Brazil, the state of Pará has the highest percentage of deaths among elderly population $(41.4 \%)$. Elder women older than 80 years has the highest percentage of deaths $(36,5 \%)$, while deaths are more frequent in men aged between 70 to 79 years $(36.5 \%)$. Diseases of the circulatory system $(27.7 \%)$ are the main cause of mortality in the elderly patients, followed by diseases of the respiratory system $(22.2 \%)$. In the subgroup analysis by sex, the main causes of death are endocrine, nutritional and metabolic diseases in the female individuals $(4.5 \%)$ and diseases of the gastrointestinal tract in the male $(8.3 \%)$. Pathologies involving the circulatory and respiratory system are responsible for the majority of deaths among elderly participants in the State of Pará. This result is similar to analysis from other Brazilian States, for the same age range. Public policies, based on prevention and health promotion, as well as expanding access to medical assistance, became fundamental for a better aging process.

Key-Words: Hospital Mortality; Health of the Elderly; Cause of Death; Health Unic System.

\section{Referências}

BEARD, J.R.;OFFICER, A.; CARVALHO, I.A.; SADANA, R.; POT, A.M.; MICHEL, J.P.; LLOYD-SHERLOCK, P.; EPPING-JORDÂNIA, P.; PEETERS, G.; MAHANANI, W.R; THIYAGARAJAN, J.A.; CHATTERJI, S. The World report on ageing and health: a policy framework for healthy ageing. : a policy framework for healthy ageing. The Lancet, [s.l.], v. 387, n. 10033, p. 2145-2154, maio 2016. Doi: 10.1016/s0140-6736(15)00516-4.

BLOOM, D. E.. 7 Billion and Counting. Science, Washington, v. 333, n. 6042 , p. 562 569, 28 jul. 2011. American Association for the Advancement of Science (AAAS). Doi: 10.1126/science.1209290.

BORIM, F. S. A.; FRANCISCO, P. M. S. B.; NERI, A. L. Sociodemographic and health factors associated with mortality in community-dwelling elderly. Revista de saude publica, São Paulo, v. 51, p. 42, 2017. Doi: 10.1590/s1518-8787.2017051006708.

CARVALHO, M. H. R.; CARVALHO, S.M.R.; LAURENTI, R.; PAYÃO, S.L.M. Tendência de mortalidade de idosos por doenças crônicas no município de Marília-SP, Brasil: 1998 a 2000 e 2005 a 2007. Epidemiologia e Serviços de Saúde, Brasília, v. 23, n. 2, p. 347-354, 2014. Doi: 10.5123/S1679-49742014000200016.

CORDEIRO, P.; MARTINS, M. Mortalidade hospitalar em pacientes idosos no Sistema Único de Saúde, região Sudeste. Revista de Saúde Pública, São Paulo, v. 52, p. 69, 2018. Doi: 10.11606/S1518-8787.2018052000146.

DEPARTAMENTO DE INFORMÁTICA DO SUS. CID 10 - DATASUS. Disponível 
em: <http://datasus.saude.gov.br/sistemase-aplicativos/cadastros-nacionais/cid-10>. Acesso em: 23 out. 2019.

FREITAS, E. V; PY, L. Tratado de Geriatria e Gerontologia. 3. ed. Rio de Janeiro: Guanabara Koogan, 2013.

JÚNIOR, R.T. ; LOFFREDO, L.C.M.. Mortalidade de idosos em município do Sudeste brasileiro de 2006 a 2011. Ciência \& Saúde Coletiva [online], Rio de Janeiro, v. 19, n. 3, p. 975-984, mar. 2014. FapUNIFESP (SciELO). Doi: 10.1590/1413-81232014193.10482012.

KERNKAMP, C. L.; COSTA, C.K.F.; MASSUDA, E.M.; SILVA, E.S.; YAMAGUCHI, M.U.; BERNUCI, M.P. Perfil de morbidade e gastos hospitalares com idosos no Paraná, Brasil, entre 2008 e 2012. Caderno de Saúde Pública [online], Rio de Janeiro, v. 32, n. 7, 2016. Doi: 10.1590/0102-311X00044115.

KUMAR, V.; ABBAS, A. K.; ASTER, J. C. Robbins and Cotran Pathologic Basis of Disease. Rio de Janeiro: Elsevier, 2016.

LAURENTI, R.; JORGE, M.H.P.M.; GOTLIEB, S.L.D. Perfil epidemiológico da morbi-mortalidade masculina. Ciência \& Saúde Coletiva, Rio de Janeiro, v. 10, n. 1, p. 35-46, 2005. Doi: 10.1590/S141381232005000100010 .

LOZANO, R. et al. Global and regional mortality from 235 causes of death for 20 age groups in 1990 and 2010: A systematic analysis for the Global Burden of Disease Study 2010. The Lancet, [s.l.], v. 380, n. 9859, p. 2095-2128, 2012. Doi: 10.1016/S01406736(12)61728-0.

MANSUR, A. D. P.; SOUZA, M. F. M.; TIMERMAN, A.; AVAKIAN, D.; ALDRIGHI, J. M.; RAMIRES, J. A. F. Tendência do risco de morte por doenças circulatórias, cerebrovasculares e isquêmicas do coração em treze estados do Brasil, de 1980 a 1998. Arquivos Brasileiros de Cardiologia, São Paulo, v. 87, n. 5, p. 641-648, nov. 2006. Doi: 10.1590/ S0066-782X2006001800015.
MCCORMICK, B.; PEARSON, M.; WHITE, J.. Hospital mortality rates and place of death. Journal of Public Health, [s.l.], p. 800805, 24 dez. 2015. Oxford University Press (OUP). Doi: 10.1093/pubmed/fdv188.

MENDES, J. D.; SALA, A. Perfil da morbidade das internações masculinas no Estado de São Paulo. Boletim Epdemiológico Paulista (Online), São Paulo, v. 8, n. 93, p. 22-31, 2011. Disponível em: <http://periodicos.ses.sp.bvs.br/scielo.php?script $=$ sci arttext\&pid=S1806-42722011000900004\&ln $\mathrm{g}=\mathrm{pt} \& \mathrm{nrm}=$ iso\&tlng $=\mathrm{pt}>$.

MENDES, J. D. V. Perfil da Mortalidade de Idosos no Estado de São Paulo em 2010. Boletim Epdemiológico Paulista (Online), São Paulo, v. 9, n. 99, p. 33-49, mar. 2012. Diponível em: < http://periodicos.ses.sp.bvs. br/scielo.php?script=sci_arttext\&pid=S180642722012000300003\&lng=pt\&nrm=iso.\#: :t ext $=0 \% 20$ n $\%$ C3\%BAmero $\% 20$ absoluto $\% 20$ de $\% 20 \%$ C 3\%B3bitos, $2000 \% 20$ para $\% 20$ $364 \% 20 \mathrm{em} \% 202010>$.

MINISTÉRIO DA SAÚDE. Dados de Morbimortalidade Masculina no Brasil. Editora MS/CGDI, 2017. Disponível em: <https://portalarquivos2.saude.gov.br/images/pdf/2018/ fevereiro/19/Folder---dados-de-morbimortalidade-masculina-no-Brasil.pdf $>$. Acesso em: 28 out. 2019 .

MOREIRA, M. C. N.; GOMES, R.; RIBEIRO, C. R. "Are men coming to the clinic now?!" Healthcare strategies for men. Cadernos de Saúde Pública, Rio de Janeiro, v. 32, n. 4, p. 1-10, 2016. Doi: 10.1590/0102-311X00060015.

MOROSINI, S.; MARQUES, A. P. O.; LEAL, M. C. C.; MARINO, J. G.; MELO, H. M. A. Custo e tempo de permanência hospitalar de idosos residentes em Recife - PE. Geriatrics, Gerontology and Aging, v. 5, n. 2, p. 91-98, 2011. Diponível em: <https://cdn.publisher. gn1.link/ggaging.com/pdf/v5n2a08.pdf>.

OLIVEIRA, T. C. DE; MEDEIROS, W. R.; LIMA, K. C. DE. Diferenciais de mortalidade por causas nas faixas etárias limítrofes de idosos. Revista Brasileira de Geriatria e Ge- 
rontologia, Rio de Janeiro, v. 18, n. 1, p. 8594, 2015. Doi:10.1590/1809-9823.2015.14203

PARENTE, A. S.; PARENTE, A. S.; VIEIRA, M. C. A. Perfil de morbidade e custos hospitalares com idosos no estado de Pernambuco. Revista Kairós : Gerontologia, São Paulo, v. 21, n. 1, p. 71-91, 2018. Doi: 10.23925/2176-901X.2018v21i1p71-91.

RODRIGUES, P. C. O.; IGNOTTI, E.; ROSA, A. M.; HACON, S. S. Distribuição espacial das internações por asma em idosos na Amazônia Brasileira. Revista Brasileira de Epidemiologia, v. 13, n. 3, p. 523-532, 2010. Disponível em: <https://www.scielo.br/pdf/ rbepid/v13n3/15.pdf $>$.

SANTOS, F. D. S.; LIMAJÚNIOR, J. O Idoso e o Processo de Envelhecimento: Um estudo sobre a qualidade de vida na terceira Idade. Id Online Revista de Psicologia, v. 8, n. 24, p. 34-55, nov. 2014. Doi: 10.14295/idonline. v8i24.300.

STEVENS, G. A. et al. Diretrizes para o relato preciso e transparente de estimativas de saúde: a Declaração GATHER. Epidemiologia e Serviços de Saúde : Revista do Sistema Único de Saúde do Brasil, Brasília, v. 26 , n. 1, p. 215-222, 2017. Doi: 10.5123/ s1679-49742017000100023.

TEIXEIRA, T.I.; OLIVEIRA, N.G.; THEODORO, H.; BRANCO, C.S. Consumo de Lipídeos e sua contribuição nos Distúrbios Metabólicos em Mulheres Adultas e Idosas da Serra Gaúcha, Sul do Brasil. Revista Brasileira de Obesidade, Nutrição e Emagrecimento, São Paulo, v. 13, p. 299-307, 2019. http://www.rbone.com.br/index.php/rbone/ article/view/942.

TELAROLLI JÚNIOR, R.; LOFFREDO, L. DE C. M. Mortalidade de idosos em município do Sudeste Brasileiro de 2006 a 2011. Ciência e Saúde Coletiva, v. 19, n. 3, p. 975-984, 2014. Doi: 10.1590/1413-81232014193.10482012.

TORTORA, G. J.; DERRICKSON, B. Princípios de Anatomia e Fisiologia. 14. ed. Rio de janeiro: Guanabara Koogan, 2016.
Autor para correspondência

Matheus Sodré de Araújo

Rua Chico Mendes, Quadra 23, Lote 12

Bairro Bom Planalto, Marabá- PA

E-mail: matheussodredearaujo@gmail.com 